\title{
Servicios ecosistémicos de regulación que aporta el guacano (Morella pavonis) en Chapisca, valle de Lluta, región de Arica y Parinacota, Chile
}

\author{
Ecosystem regulatory services provided by the Guacano (Morella pavonis) \\ in Chapisca, Lluta Valley, Arica and Parinacota region, Chile
}

\author{
Daniela Bahamondes $^{1 *}$, Paola Moraga ${ }^{2}$, Eliana Belmonte ${ }^{3}$
}

\begin{abstract}
RESUMEN
Los servicios ecosistémicos son los beneficios que las sociedades obtienen de los ecosistemas. Este concepto permite hacer más explícita la interdependencia del bienestar humano y el mantenimiento del adecuado funcionamiento de los ecosistemas. En relación con su clasificación, se utiliza la propuesta de Evaluación de los Ecosistemas del Milenio (MEA) que divide los servicios ecosistémicos en cuatro grupos: servicios de provisión, de soporte, culturales y de regulación. Este último son funciones no consuntivas que generan condiciones ecosistémicas que no pasan por procesos de transformación o manufactura. Es por esto que surgió la necesidad de examinar e identificar los servicios ecosistémicos de regulación (SER) que aporta la especie arbórea y dioica Morella pavonis a la localidad de Chapisca, ubicada a $60 \mathrm{~km}$ de Arica en el valle de Lluta, región de Arica y Parinacota. En primera instancia, se identificaron in situ las islas vegetacionales de Morella pavonis y la cobertura vegetacional asociada a ella. En una segunda instancia, utilizando el sistema internacional de información geográfica (SIG) se determinaron los servicios ecosistémicos de regulación que aporta la especie Morella pavonis a la región de Arica y Parinacota. Como resultado del estudio se identificó un SER relacionado directamente con la temperatura ambiental. Con el tiempo la temperatura ha ido en aumento, condición inversamente proporcional a la abundancia relativa de la especie arbórea, que ha disminuido en alrededor de un $62 \%$ la superficie en el ensamble ecológico. Se plantea revisar su actual estado de conservación y generar un nuevo estado del arte para la protección y conservación de la especie.
\end{abstract}

Palabras clave: Vulnerabilidad, cobertura vegetacional, servicio ecosistémico, especie nativa y bienestar humano.

\begin{abstract}
Ecosystem services are the benefits that societies obtain from ecosystems. This concept allows making more explicit the interdependence of human well-being and the maintenance of the proper functioning of ecosystems. Regarding their classification, the Millennium Ecosystem Assessment proposal (MEA) is used, which classifies ecosystem services into four groups; Provision, support, cultural and regulation supplies, the latter being non-consumptive functions that generate ecosystem conditions that do not undergo transformation or manufacturing processes. This is why there was a need to examine and identify the ecosystem services (IES) provided by the tree and dioecious species, Morella pavonis to the town of Chapisca, located $60 \mathrm{~km}$ from Arica through the Lluta valley in the region from Arica and Parinacota. In the first instance, the vegetative islands of Morella pavonis and the associated vegetation cover were identified in situ; In a second instance, using the international geographic information system (QGIS), the regulatory ecosystem services provided by the Morella pavonis species to the Arica and Parinacota region were determined. As a result of the study, an SER was directly related to the ambient temperature. Over time the temperature has been increasing, an environmental condition inversely proportional to the relative abundance of the tree species, decreasing in around $62 \%$ its surface in the ecological assembly. It is proposed to review its current state of conservation and generate a new state of the art for the protection and conservation of the species.
\end{abstract}

Keywords: vulnerability, vegetation cover, ecosystem service, native species and human well-being.

\footnotetext{
Pontificia Universidad Católica de Chile, Facultad de Agronomía e Ingeniería Forestal. Santiago, Chile.

Agrupación Social, Cultural y del Medio Ambiente PALBAS. Arica, Chile.

Universidad de Tarapacá, Facultad de Ciencias, Departamento de Biología. Arica, Chile.

* Autor por correspondencia: dlbahamondes@uc.cl.
}

Fecha de Recepción: 1 de Enero, 2020.

Fecha de Aceptación: 03 de marzo, 2020. 


\section{Introducción y revisión bibliográfica}

Los conceptos de servicios ecosistémicos y biodiversidad se examinan desde una perspectiva sistémica. Es decir, que se pueden considerar como las contribuciones directas e indirectas de las estructuras y funciones del ecosistema (Muller y Burkhard, 2012). Esta definición pone énfasis en las condiciones biofísicas cambiantes dentro de los ecosistemas, así como en las interacciones (procesos) entre estas y sus componentes bióticos (especies). "Los servicios ecosistémicos incluyen el suministro de insumos de los ecosistemas, la capacidad de asimilación del medio ambiente y la prestación de la biodiversidad". (OCDE, 2013). Estos comprenden servicios de aprovisionamiento, servicios de soporte, servicios culturales y servicios de regulación que mantienen las condiciones de vida en la Tierra (Balvaner, 2012; Rodríguez et al., 2016). En otras palabras, el concepto de servicios ecosistémicos permite hacer un vínculo explícito entre el estado y funcionamiento de los ecosistemas (sistemas ecológicos) y el bienestar humano (sistemas sociales), y contribuir a orientar (o reorientar) el rumbo del manejo de la base biofísica que debe sustentar el bienestar humano en escenarios de cambio ambiental. Esta relación puede ser directa o indirecta, y los seres humanos pueden o no estar conscientes de su existencia.

Al considerar la información analizada, surgió la necesidad de examinar e identificar los servicios ecosistémicos de regulación (SER) que aportan las especies arbóreas, ya que la vegetación -y en especial árboles y arbustos-, tiene la capacidad de mejorar la calidad del aire a través de dos procesos: la absorción de gases en el desarrollo de la fotosíntesis y la fijación en la superficie del follaje de partículas de polvo suspendidas (MEA, 2005). Los contaminantes son absorbidos por las estomas del follaje y atrapados en los espacios intracelulares

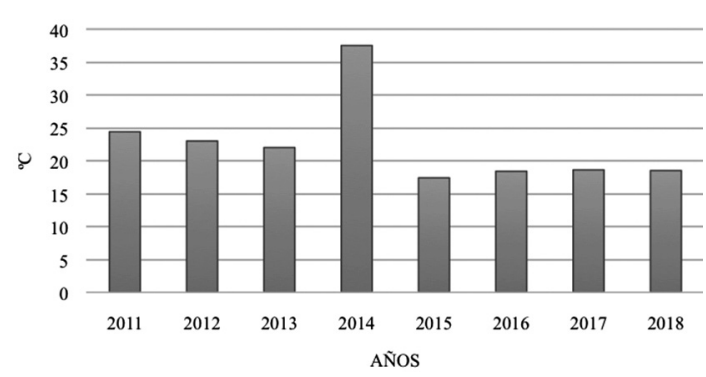

Figura 1. Variación de temperatura $\left({ }^{\circ} \mathrm{C}\right)$ en el sector de Chapisca. de las hojas para posteriormente incorporarlos a los procesos metabólicos de la planta o árbol. La vegetación realiza este proceso a través de su masa foliar, por consiguiente, esta capacidad es mayor para los árboles, por sobre los arbustos y pastizales, ya que mantienen una mayor área foliar (Escobedo et al., 2008; Dobbs et al., 2011).

Otra variable importante en el monitoreo de la densidad del bosque es la cobertura arbórea (COB), que regula la cantidad de luz penetrante y controla ciertos procesos ecológicos, reflejando y absorbiendo radiaciones que pueden ser nocivas para la salud humana y proporcionando sombra que aporta al bienestar antrópico (Escobedo y Chacalo, 2008). Además, la radiación solar (Figura 3) a nivel de suelo puede generar altas temperaturas que causen incomodidad a los seres humanos, (Figura 1 y 2 ) además de limitación en el uso de espacio, y que en consecuencia prefieran un lugar con las condiciones térmicas de una zona de confort aceptable, antes que otros que no lo permiten (Guillén-Mena y Orellana, 2016).

La especie Morella pavonis, perteneciente a la familia Myricaceae y conocida comúnmente como Guacano, es parte de la biodiversidad de la región de Arica y Parinacota, específicamente en la localidad de Chapisca, valle de Lluta, y es la única representante nativa de un género cosmopolita con alrededor de 50 especies (Rodríguez et al., 2003). Es un árbol dioico, de copa globosa, que alcanza $12 \mathrm{~m}$ de alto y tronco sinuoso de hasta $80 \mathrm{~cm}$ de diámetro (MMA, 2007). La corteza es de color café grisáceo rugosa con fisuras transversales y protuberancias suberosas.

\section{Materiales y métodos}

En la actualidad las variables de densidad vegetal pueden ser obtenidas mediante datos espectrales a través de sensores en plataformas satelitales.

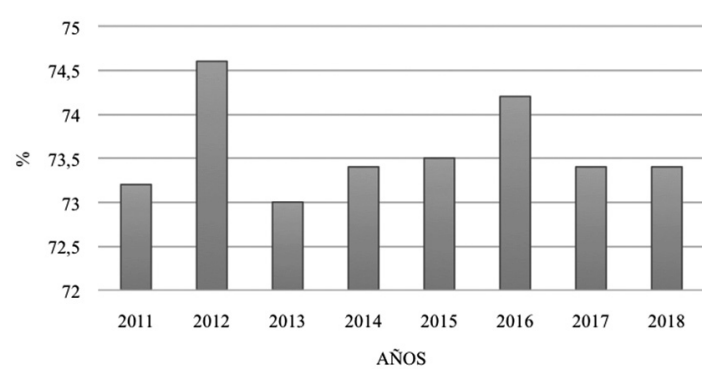

Figura 2. Humedad relativa (\%) sector de Chapisca. 


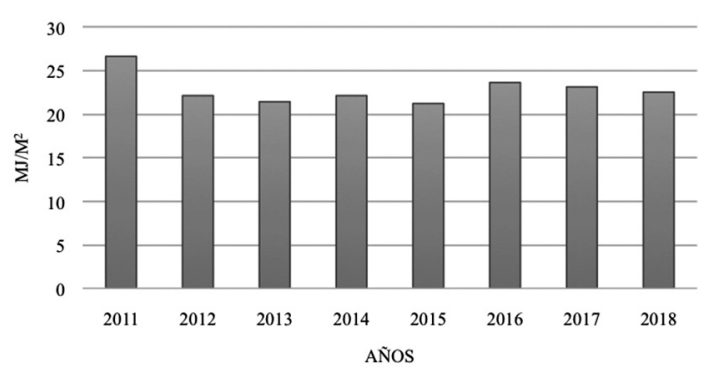

Figura 3. Radiación solar $\left(\mathrm{Mj} / \mathrm{m}^{2}\right)$ en el sector de Chapisca.

Es por ello que de manera complementaria, y de acuerdo a los estándares exigidos en los procesos de investigación, se deben utilizar metodologías adecuadas a las necesidades de hoy en día. Los sistemas de información geográfica de software libre son esenciales para los procesos que impliquen el análisis de formatos de datos ráster y vectoriales, a través de bibliotecas y bases de datos, que es donde radican sus grandes potencialidades. El análisis de la cobertura arbórea de Morella pavonis en el sector de Chapisca, se realiza desde una mirada científica y con metodologías exploratorias cualitativas y cuantitativas en el territorio, que se complementan con las nuevas tecnologías a través de programas satelitales como el software QGIS en el cual basamos esta investigación y que permitió determinar el servicio ecosistémico de regulación que otorga Morella pavonis.

\section{Recopilación de información secundaria}

Debido a la escasa bibliografía existente sobre los diferentes tópicos que aborda esta investigación, se hizo necesariorealizar una recopilación de información secundaria obtenida de documentos científicos y sitios web oficiales. Posteriormente se realizó una clasificación de la información recopilada a través de temáticas asociadas a la investigación: características morfológicas de la especie arbórea, ciclo de vida de la especie arbórea, usos de la especie arbórea, características y datos meteorológicos del área de investigación, con el fin de identificar los principales factores asociados a este estudio. En cada documento se consideraron las características generales, relevando las sinergias y acoplamientos entre ellos, lo que luego fue utilizado como parte de la discusión.

\section{Exploración en el territorio}

De acuerdo a los datos obtenidos del análisis de la información secundaria, se determinó que la especie en estudio se encuentra a lo largo de la ruta A15 a 61,5 kilómetros de la ciudad de Arica en la región de Arica y Parinacota, específicamente entre las localidades de Chapisca y Sora.

Dentro de la zona establecida, la exploración se realizó desde las zonas más altas a las más bajas, utilizando las vías de acceso público y en algunos casos a través de accesos privados, previo consentimiento de sus dueños. Se definió la ubicación georeferencial (GPS) y se hizo el registro fotográfico de las áreas donde se visualizó la especie.

\section{Identificación de islas vegetacionales de Morella pavonis}

En primera instancia, fue necesario recurrir a la ficha técnica de la especie obtenida en la recopilación de información secundaria, así como a las áreas determinadas en la exploración del territorio. Con ambas informaciones se realizó una descripción del área basándose en la riqueza de especies (flora asociada) que componen la isla vegetacional donde se encuentra Morella pavonis. Se identificó su ciclo biológico y sus características morfológicas como sus lenticelas, altura, forma y tipo de hoja y su estado. Cada observación fue registrada en cuaderno de campo y a través de 552 fotografías. Además, se hicieron registros, tanto digitales como manuales de georreferencia (GPS).

\section{Análisis de Servicios Ecosistémicos de regulación a través del Sistema de Información Geográfica (SIG) en QGIS}

Los datos fueron analizados con técnicas geoespaciales en el software QGIS. Toda la información recolectada tuvo un sistema de referenciación exacta, correspondiente a un sistema de coordenadas Universal Transversal de Mercator (UTM).

Las cartografías para el desarrollo de esta etapa fueron proporcionadas a través de las imágenes satelitales Landsat (5 ETM+) entre los años 1980 y 2018, con un cubrimiento específico del sector de Chapisca. Se accedió a esta información por medio del Instituto Nacional de Pesquisas Espaciais (INPE).

El procesamiento de las imágenes satelitales consta de dos (2) fases: entrenamiento y asignación. En la fase 1, los estudiantes realizaron un 
reconocimiento general del área definida para esta investigación, determinando patrones de formas y colores relacionados con una clase que permita identificar a Morella pavonis, entrenando el conjunto de píxeles a cada clase encontrada. Se desarrolla una descripción numérica de las características espectrales de las bandas que discriminan los grupos de píxeles pertenecientes a una misma clase, mediante la generación de sus firmas espectrales (Arango et al., 2005). En la fase 2 , se asignó una lista de clases o nombres a cada patrón observado, generando mediante algoritmos una clasificación general de la imagen.

Finalmente, para visualizar la dinámica de la permanencia de Morella pavonis y reconocer servicios ecosistémicos entregados por la especie en el territorio, se realizó un análisis estadístico descriptivo espacio-temporal, por medio de una clasificación representada en unidades vectoriales para su posterior representación gráfica. Esto permitirá evidenciar la pérdida de la especie en un tiempo determinado, así como la cobertura vegetacional. Esta última es clave para el proceso de reconocimiento e identificación de los servicios ecosistémicos de regulación, los cuales serán proporcionados visualmente a través de imágenes proyectadas, producto del análisis desarrollado por el software QGIS.

\section{Resultados y discusión}

\section{Recopilación de información secundaria}

La especie Morella pavonis es endémica de la región de Arica y Parinacota y a pesar de esta característica, los estudios sobre ella son escasos. La última información publicada que describe sus características fue el año 1985 por Belmonte, mientras que su ficha técnica fue actualizada el año 2007 por el Ministerio del Medio Ambiente, situación que llevó a considerar a esta especie arbórea en estado de vulnerabilidad. Dentro de sus características morfológicas se destaca que puede llegar a medir hasta $12 \mathrm{~m}$ de alto, su copa es globosa y las hojas simples, alternadas $\mathrm{y}$ abundantes, lo que puede generar una sombra, ayudando a contrarrestar las altas temperaturas en la zona donde se encuentre. En relación con el ciclo de vida, la información bibliográfica indica que la floración de la especie se desarrolla en verano, puntualmente cuando las temperaturas ambientales aumentan. En cuanto a los usos que se le dan a la especie, Belmonte (1985) indica que es utilizada para fabricación de herramientas. Mientras que Ugarte (2004) señala que Morella pavonis ha sido usada a lo largo de los años en la construcción de viviendas debido a su firmeza.

Con respecto a los datos meteorológicos de los últimos ocho años, sostienen que existe una variabilidad de la temperatura superficial entre los 18 y $25^{\circ} \mathrm{C}$ en promedio, exceptuando el año 2014 , que registra un promedio anual de $37,5^{\circ} \mathrm{C}$ (INIA, 2019). Esto, según las características climáticas del extremo norte de Chile, coincide con la tendencia de los datos analizados en la investigación.

\section{Exploración en el territorio}

Al realizar la exploración del territorio por la ruta A15 encontramos la mayor concentración de especies en la localidad de Chapisca. Sin embargo, el primer grupo de individuos fue observado en el poblado de Molinos, mientras que el último en la localidad de Sora (Figura 4).

\section{Identificación de islas vegetacionales de Morella pavonis}

Los distintos análisis de las muestras reflejaron que no existe un patrón de comportamiento para su ciclo biológico. Se desprende que las especies que se encontraban más cerca del cauce hidrológico natural presentaban un ciclo de desarrollo normal de acuerdo con los ciclos de vida de la familia Myricaceae, mientras que las especies que se hallaban en zonas parceladas de desarrollo agrícola, alejadas del cauce hidrológico natural, mostraban una mayor presencia de la etapa vegetativa del ciclo biológico de Morella pavonis.

Del análisis de las distintas muestras tomadas en el sector de Chapisca se pudo determinar la flora asociada a Morella pavonis, la cual se detalla en la Tabla 1.

\section{Análisis de Servicios Ecosistémicos de regulación a través del Sistema de Información Geográfica (SIG) en QGIS}

Existe una tendencia decreciente en los patrones de ocupación de la especie Morella pavonis desde el año 1980 hasta el 2018. Al analizar los usos y 


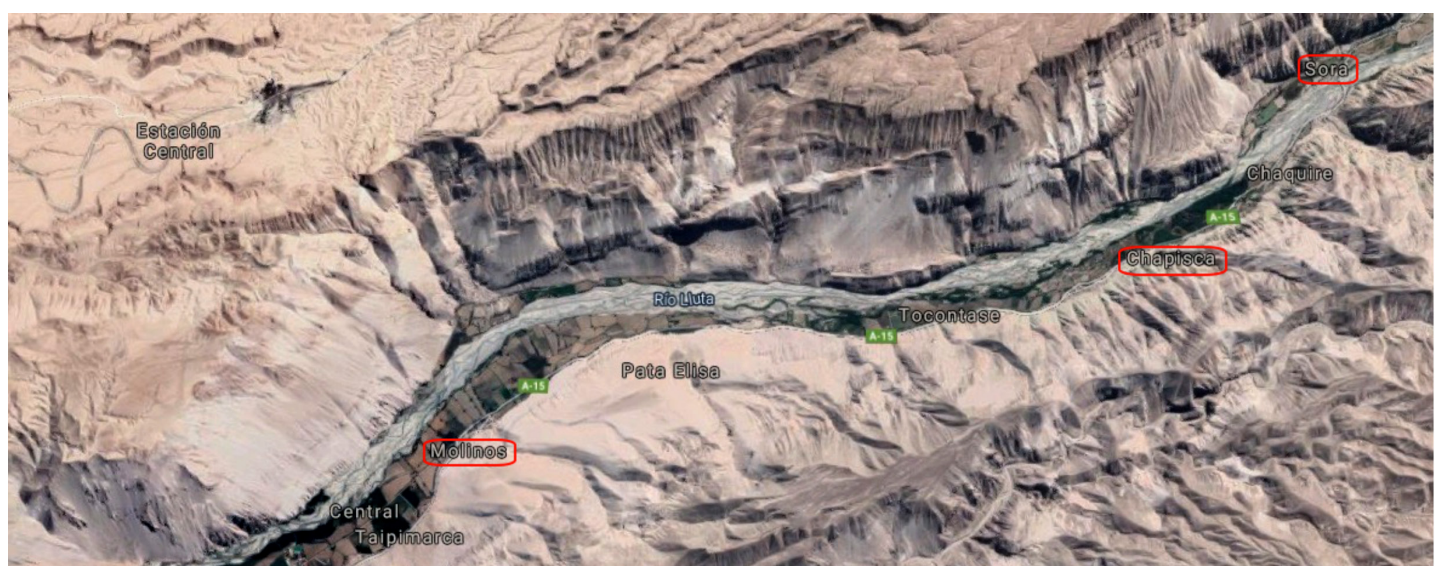

Figura 4. Localidades de la región de Arica y Parinacota donde se encuentran las islas vegetacionales de Morella pavonis. Fuente: Google earth ${ }^{\circledR}$

Tabla 1. Flora asociada a

Morella pavonis en el sector de Chapisca.

\begin{tabular}{ll}
\hline \multicolumn{1}{c}{ Nombre científico } & Nombre Común \\
\hline Baccharis alnifolia & Chilca \\
Cortaderia speciosa & Cola de zorro \\
Distichlis spicata & Grama salada \\
Equisetum giganteum & Cola de caballo \\
Schoenoplectus americanus & Junquillo \\
\hline
\end{tabular}

coberturas que la sustituyen, se evidencia que los espacios de especies provenientes del grupo Morella pavonis son sustituidos en más de 51 hectáreas (ha) por zonas de cultivos y/o terrenos de secano sin cultivos. A pesar de su disminución, de igual manera su cobertura arbórea genera sombra, lo que permite regular la temperatura de la zona (Figura 5).

Los datos mostrados anteriormente reflejan una disminución de la superficie de Morella pavonis en un $62 \%$ desde el año 1980 hasta 2018. Actualmente su cobertura vegetacional está restringida a 31 hectáreas (Figura 6).

Dentro de los servicios ecosistémicos que los pobladores de la localidad de Chapisca obtienen de Morella pavonis, se encuentran los de soporte, con el uso ancestral de su madera para la construcción de postes de vivienda (Ugarte, 2004); y los de provisión, ya que esta especie ha sido utilizada para la fabricación de herramientas y como combustible (Belmonte, 1985; MMA, 2007). Esta situación ha generado que muchas poblaciones de Morella pavonis hayan reducido su número de especies por población, lo cual llevó a que en el año 2008 se declarara su estado de conservación como vulnerable bajo la Ley General de Bases del Medio Ambiente $N^{\circ}$ 19.300. Esto puede ser comparable con los registros inéditos incorporados en esta investigación que dejan de manifiesto que desde el año 1980 al 2018 hubo una reducción de la superficie de la especie (Figura 6).

De acuerdo con la Ley General de Bases del Medio Ambiente $\mathrm{N}^{\circ} 19.300$ y basándose en el trabajo realizado por De Lima et al. (2016), quienes hacen mención a la existencia de otros servicios ecosistémicos que se obtienen sin la invasión de la especie, se considera que los servicios ecosistémicos de regulación, tales como la cobertura arbórea, son los que se deben valorar en función de las características propias de Morella pavonis, por cuanto no serían perjudiciales para la manutención de la especie.

Por otra parte, los registros bibliográficos del desarrollo de Morella pavonis en la región de Arica y Parinacota son escasos y no tienen concordancia con el ciclo biológico del árbol en relación con la caracterización del suelo y del agua del territorio en estudio. Sin embargo, la información obtenida se considera inédita y pasa a ser el puntapié inicial para futuras investigaciones científicas.

\section{Conclusión}

Luego de analizar las características morfológicas propias de la especie y de explorar el territorio, se puede determinar que los servicios ecosistémicos de regulación que proporciona 


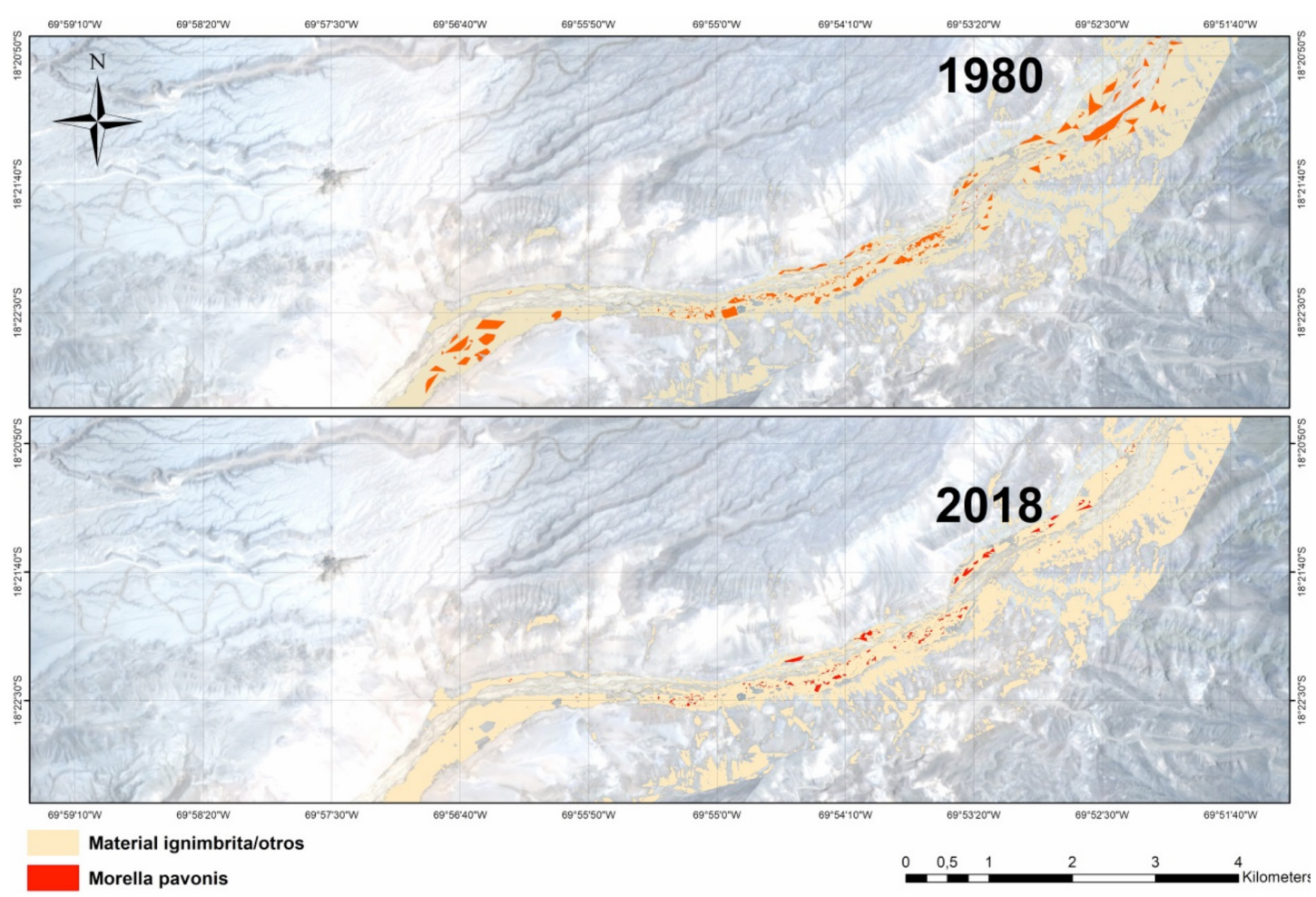

Figura 5. Mapa comparativo de la población de Morella pavonis entre los años 1980 y 2018, en la localidad de Chapisca y Sora, región de Arica y Parinacota. Fuente: QGIS® Geographic Information System.

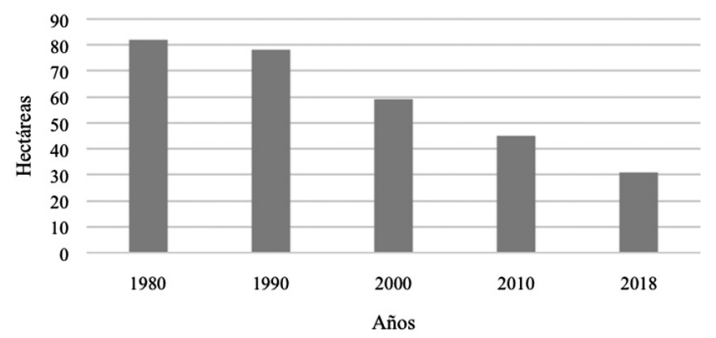

Figura 6. Cobertura (Has) de Morella pavonis comprendida en 38 años, en la localidad de Chapisca, región de Arica y Parinacota.

Morella pavonis a los pobladores de la localidad de Chapisca, valle de Lluta, son sombra y reducción de temperaturas extremas. Sin embargo, al comparar datos de años anteriores con la nueva base de datos creada para esta investigación, se concluyó que la cantidad de individuos a lo largo de los años ha disminuido, lo cual ha ocasionado que la cobertura arbórea sea menor. Esto debido al uso de los servicios ecosistémicos de aprovisionamiento y soporte, los cuales han llevado a que en la actualidad existan menos poblaciones de Morella pavonis.

Es por ello que en un futuro se considera fundamental diseñar estrategias para que los pobladores comprendan la importancia de esta especie arbórea en el sector, ya que no solo es un árbol endémico de la zona que puede generar recursos para la especie humana, sino que también está en estado de vulnerabilidad. Se estima que se pueda elaborar una nueva base de datos que permita dejar registro actualizado, a fin de determinar la abundancia de Morella pavonis en el sector de Chapisca y sus alrededores (Molino y Sora), y contribuir así a la preservación de la biodiversidad de la región de Arica y Parinacota. 


\section{Literatura Citada}

Arango, G.M.; Branch, B.; Botero, F.

2005. Clasificación no supervisada de coberturas vegetales sobre imágenes digitales de sensores Remotos: -LandsatETM+II. Revista Facultad de Agronomía, 58(1): 2611-2634.

Balvanera, P.

2012. Los servicios ecosistémicos que ofrecen los bosques tropicales. Ecosistemas, 21(1-2): 136-147.

Belmonte, E.

1985. Simposio sobre la flora nativa arbórea y arbustiva de Chile amenazada de extinción. Idesia, 9: 63-64.

Benoit, I.

1989. Libro rojo de la flora terrestre de Chile. CONAF. Santiago, Chile. 157 p.

De Lima, S.; Rojas, M.; Méndez, J.; Salazar, K; Salmerón, A. 2016. Servicios ecosistémicos de regulación que benefician a la sociedad y su relación con la restauración ecológica. Boicenosis, 31: 80-92.

Dobbs, C.; Escobedo, F.; Zipperer, W.C.

2011. Aframework for developing urban forest ecosystem services and godos indicators. Landscape and Urban plannign, 99: 196-206.

Escobedo, F.; Chacalo, A.

2008. Estimación preliminar de la descontaminación atmosférica por el arbolado urbano de Ciudad de México. INCI, 33(1): 29-32.
Guillén-Mena, V.; Orellana, D.

2016. La influencia de la vegetación arbórea urbana para disminuir el nivel de polución y alcanzar el confort climático. Revista de la Facultad de Ciencias Químicas, Volumen especial: 67-75.

Muller, F.; Burkhard, B.

2012. The indicator side of ecosystem services. Ecosyst. Serv., 1(1): 26-30.

OCDE.

2013. Evaluaciones de la OCDE sobre el desempeño ambiental: OECD Publishing. París, Francia. 192 p.

Rodríguez, R.; Quezada, M.

2003. Myricaceae. En: Marticorena, C.; R. Rodríguez (eds.). Flora de Chile. Vol. 2. Santiago, Chile. pp. 62-63.

Rodríguez, L.; Guretti, G.; Garegnani, G.; Grilli, G.; Pastorella, F.; Paleto, A.

2016. La valoración de los servicios ecosistémicos en los ecosistemas forestales: un caso de estudio en Los Alpes Italianos. Bosque, 37(1): 41-52.

Ugarte, $\mathrm{M}$.

2004. Identificación de especies de madera en postes de vivienda en sitios del valle de Lluta. Chungara, Volumen Especial: 1015-1022. 
